\title{
SpaceOAR to improve dosimetric outcomes for monotherapy high-dose-rate prostate implantation in a patient with ulcerative colitis
}

\author{
Michael Trager, MS, Benjamin Greenberger, MD, Amy S Harrison, PhD, James Keller, PhD, Robert B Den, MD \\ Department of Radiation Oncology, Thomas Jefferson University Hospital, Philadelphia, PA, USA
}

\begin{abstract}
High-dose-rate (HDR) brachytherapy is an attractive option for patients receiving definitive radiation therapy for prostate cancer with decreased overall dose to the pelvis. However, ulcerative colitis increases rectal toxicity risk and may be a contraindication. A synthetic hydrogel, SpaceOAR (Augmentix Inc., Waltham, MA, USA), can facilitate the use of HDR brachytherapy for patients where rectal toxicity is a limiting factor.

SpaceOAR gel $(13.19 \mathrm{cc})$ was utilized in a monotherapy HDR prostate treatment with Ir-192 under transrectal ultrasound guidance, with the intention of decreasing rectal dose. SpaceOAR gel was inserted transperineally into the patient 18 days prior to the procedure.

The HDR brachytherapy procedure was tolerated without incident. All planning constraints were met, and the following dosimetry was achieved: Prostate $-\mathrm{V}_{100 \%}=97.3 \%, \mathrm{~V}_{150 \%}=35 \%, \mathrm{~V}_{200 \%}=14.5 \%$; Urethra $-\mathrm{V}_{118 \%}=0 \%$; Rectum $-\mathrm{D}_{2 \mathrm{cc}}=51.6 \%, \mathrm{~V}_{75 \%}=0 \mathrm{cc}$. The rectum-catheter spacing was on average between 6-8 mm. Average spacing for our 10 most recent patients without SpaceOAR was $3 \mathrm{~mm}$. SpaceOAR did not hinder or distort ultrasound imaging or increase treatment time.

SpaceOAR successfully increases catheter-rectal wall spacing and decreases rectal dose due to improved planning capabilities, while decreasing the likelihood of rectal perforation. One application of this tool is presented to mitigate potential toxicities associated with ulcerative colitis. At five months, one week, and one day follow-up, the patient reported no bowel issues following HDR brachytherapy.
\end{abstract}

J Contemp Brachytherapy 2018; 10, 6: 577-582 DOI: https://doi.org/10.5114/jcb.2018.81001

Key words: HDR brachytherapy, HDR, interstitial, prostate HDR, SpaceOAR.

\section{Purpose}

Radiation therapy (RT) for prostate cancer is a well-established and effective treatment technique with various treatment options backed by randomized trials and single-institutional studies [1]. Although radiation modalities such as external beam radiotherapy (EBRT) and brachytherapy are effective in the management of prostate cancer, a concern regarding short and late toxicity plays a pivotal role in modality selection, dose, and duration of therapy, motivating recent advances in technique of delivery.

The ProtecT trial concluded no significant difference among the prostate-cancer-specific mortality rates in observation versus surgery or RT; however, they did note increased genitourinary (GU) toxicities and erectile dysfunction following surgery as well as increased gastrointestinal (GI) toxicities from RT [2]. Rectal toxicities are a limiting factor in RT for prostate cancer due to a high rectal dose attributed to the proximity of the prostate and rectum, with significantly higher toxicity correlated to dose to the inferior rectum $[3,4,5]$. In some patients, the degree of rectal toxicity may be a key factor governing treatment selection. Willet et al. [6] reported increased rectal toxicity associated with RT and irritable bowel disease (IBD), specifically ulcerative colitis (UC), as well as severe rectal toxicities in patients with IBD undergoing abdominopelvic irradiation. Furthermore, acute GI toxicity of grade 2 or higher is exacerbated in patients undergoing concomitant medical therapy for IBD [7]. Advances in modern RT have demonstrated a trend toward improved toxicity profiles from treatment in high-risk groups such as patients with IBD [8,9].

Although advances in image-guided radiation therapy (IGRT) and treatment technique may facilitate the use of RT in patients with a concern for rectal toxicity, modern trends in EBRT and brachytherapy application towards dose escalation and hypofractionation contribute to renewed concern regarding sparing rectal dose.
Address for correspondence: Robert B Den, MD, Department of Radiation Oncology, Bodine Center for Cancer Treatment, Thomas Jefferson University Hospital, G-301 Bodine Center, 111 S. 11th Street, Philadelphia, PA 19107, USA, phone: +1 215 955-6702, 凶 e-mail: robert.den@jefferson.edu
Received: 08.05 .2018

Accepted: 28.11 .2018

Published: 28.12.2018 
Several reports and phase III clinical trials incorporating patients of varied risk stratification have demonstrated improved biochemical control with modern dose escalated EBRT $[10,11,12,13,14,15]$. Given favorable therapeutic ratio and logistic advantages, moderate and extreme hypofractionation have been a focus of recent randomized trials of the past decade $[16,17,18,19,20]$. Although not conclusively shown in the majority of hypofractionation trials to date, there have been reports of increased rectal toxicity associated with larger dose per fraction [21].

Brachytherapy remains a curative alternative to radical prostatectomy or EBRT for early stage disease, with no significant differences in treatment results but lower rates of urinary incontinence and increased preservation of sexual function [22]. Trends in brachytherapy have similarly explored dose escalation and completion of treatment within fewer sessions with high-dose-rate (HDR) techniques [23]. Although late grade 3 rectal toxicity is rare following definitive upfront brachytherapy, well below $5 \%$ in most series, dosimetric rectal outcome has been shown to be overall improved with HDR compared to EBRT [24]. In instances of salvage brachytherapy following biochemical failure post-EBRT, a higher incidence of grade $2 / 3$ rectal toxicity has been reported in low-dose-rate (LDR) and HDR series [25]. When considering upfront treatments in the recent ASCENDE-RT trial, addition of brachytherapy boost to 78 Gy EBRT demonstrated an increase in grade 3 rectal toxicity from $3.2 \%$ to $8.1 \%$ [26]. In patients receiving HDR monotherapy (19 Gy in single fraction), Krauss et al. [27] reported grade $1 / 2$ acute and chronic GI toxicities in $12 \% / 0 \%$ and $12.1 \% / 1.7 \%$ of patients, respectively, and Prada et al. [28] reported acute grade $1 \mathrm{GI}$ toxicities up to $7 \%$, with no grade 2 acute or grade $1 / 2$ chronic GI toxicities. It is likely that further techniques designed to improve rectal dosimetry in the context of brachytherapy will continue to lead to less acute and late toxicity.

Displacement of normal tissue has long been explored as a viable strategy to improve dosimetry to organs at risk (OARs) [29]. For prostate RT, a novel technique for sparing high-dose to the anterior rectal wall is the use of a synthetic gel injection into the space between the prostate and rectum $[30,31,32,33,34]$. The feasibility and effects on toxicity of these rectal displacement gels has been reported by various investigators. Prada et al. [35] reported the successful use of a rectal wall displacement technique in single fraction HDR brachytherapy as monotherapy, with no additional toxicities and the same biochemical control at 32 months. Strom et al. [36] reported successful implementation of a polyethylene glycol (PEG) hydrogel for HDR brachytherapy plus or minus intensity-modulated radiation therapy (IMRT), with significantly increased prostate-rectal distance and significantly decreased mean rectal doses. Yeh et al. [37] similarly looked at a PEG hydrogel for HDR brachytherapy plus or minus IMRT and also reported increased prostate-rectal distance as well as low acute and chronic rectal toxicities despite aggressive dose escalation. SpaceOAR (Augmentix Inc., Waltham, MA, USA) is an FDA-approved gel, evaluated as having a benefit in reducing rectal dose, toxicity, and quality of life declines by a phase III trial with a median follow-up period of 3 years $[33,38,39]$. At our clinic, SpaceOAR is typically injected under ultrasound guidance at the conclusion of an HDR prostate procedure performed prior to external beam hypofractionated prostate RT, while patients are under general anesthesia. The use of SpaceOAR for these patients has effectively reduced high-dose to the rectum consistent with that shown in the phase III trial previously mentioned. Here, we report the potential benefit of injecting SpaceOAR prior to a monotherapy HDR prostate implantation, with the primary goal of mitigating GI toxicity due to UC.

\section{Case presentation}

A 73-year-old patient initially presented with an elevated prostate-specific antigen (PSA) of $9.94 \mathrm{ng} / \mathrm{ml}$. He received a prostate biopsy, which confirmed intermediate-risk adenocarcinoma of the prostate, GG 2 stage IIB (cT2bN0M0, Gleason score of $3+4=7$ in 3/12 cores). Brachytherapy with 19 Gy in a single fraction with SpaceOAR was chosen as monotherapy for this patient instead of EBRT due the patient's UC and concomitant medication use of azathioprine.

The SpaceOAR gel implantation was performed 18 days prior to the HDR procedure in sterile dorsal lithotomy position with an ultrasound stepper and transrectal ultrasound (TRUS). This timing was a result of logistical and scheduling considerations between the time of injection and the HDR procedure. A waiting period of at least three days before HDR is necessary to allow local gas to adequately resolve, restoring visualization of the prostate gland, which is immediately lost following the injection. Using $1 \%$ lidocaine, the perineum was numbed, followed by the prostatic neurovascular bundles via TRUS guidance. The SpaceOAR needle was then advanced to the mid prostate at midline, and a $1 \mathrm{cc}$ injection of saline was used to identify the correct position posterior to the prostate and completely anterior to the rectum. SpaceOAR was then attached and injected to completion. At Jefferson hospital, the average SpaceOAR volume injected into patients receiving hypofractionated EBRT following HDR prostate treatment is $10.5 \mathrm{cc}$ (range, 6.88-14.08 cc) as seen on magnetic resonance imaging (MRI). The SpaceOAR volume used in this case as seen on MRI was $13.19 \mathrm{cc}$ and unintentionally asymmetrically displaced slightly to the patient's left during injection. Injection volume varies with application $[30,34]$.

For the monotherapy HDR prostate brachytherapy procedure, the patient was in dorsal lithotomy position and induced under general anesthesia. A Foley catheter was inserted. Rectal preparation was verified, and perineum was prepped with betadine. A TRUS probe with a brachytherapy ultrasound stepper was inserted into the rectum, and the prostate was visualized and measured to be $39 \mathrm{cc}$. Using a template-based approach, 17 catheters were inserted from anterior to posterior prostate in a modified periphery loading technique. Catheter positions were verified in the transverse and sagittal positions and depths were confirmed. The target volume, normal tissues (rectum and urethra), and SpaceOAR were contoured by the attending physician, and real-time 

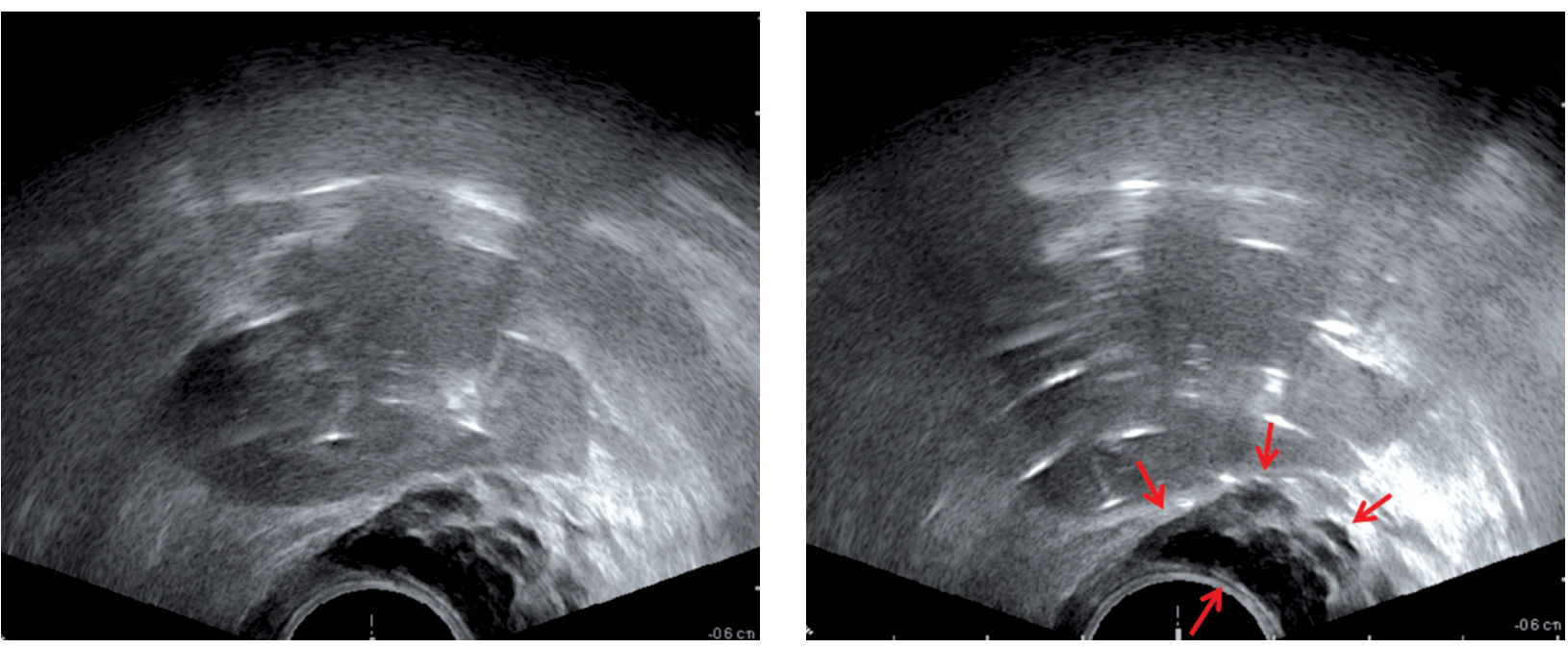

Fig. 1. Axial slices from an ultrasound image taken before (left; four anchor needles only) and after (right) complete needle implantation during an HDR brachytherapy prostate procedure post-SpaceOAR injection. The SpaceOAR gel (surrounded by red arrows in right image) and implanted needles (bright spots) are clearly visualized

inverse planning (Nucletron Oncentra ${ }^{\circledR}$ Prostate v.4.2.2.4) was performed by physics. All constraints were met as follows: Prostate $-\mathrm{V}_{100 \%}>95 \%, \mathrm{~V}_{150 \%}<35 \%, \mathrm{~V}_{200 \%}<12 \%$; Urethra - $\mathrm{D}_{\text {point } \max }<120 \%, \mathrm{D}_{10 \%}<115 \%$; Rectum $\mathrm{D}_{\text {point max }}<90 \%, \mathrm{~V}_{80 \%}<0.2$ cc [27]. After plan checks by the physician and physicists, catheters were connected to the microSelectron ${ }^{\circledR}$ v2 Ir-192 HDR afterloader (Nucletron, The Netherlands) and the treatment was delivered via Nucletron Oncentra ${ }^{\circledR}$ TCS. After delivery, catheters were removed, perineum was cleaned, and the patient was extubated. The procedure was tolerated without incident.

\section{Discussion}

The SpaceOAR is placed between the ultrasound probe and needles, thus it was hypothesized to decrease visualization of the needles due to the potential for various distorting effects seen in ultrasound such as reflection, refraction, and shadowing that occur at tissue interfaces. Consistent with vendor literature, there was no visual hindrance or distortion of the needles due to the gel and the gel was clearly visible on the ultrasound image (Figure 1). After analysis, this makes sense since the SpaceOAR gel had an average Hounsfield Unit (HU) value of 7 based on the average SpaceOAR HUs from the CT scans of patients previously treated at our institution, which is very close to water $(\mathrm{HU}=0)$ and should therefore have a similar density and acoustic impedance resulting in minimal ultrasound distortion. Maintaining satisfactory image quality validates the feasibility of utilizing SpaceOAR prior to HDR as a rectal protector for patients with UC. It is interesting to note, however, that there is a large discrepancy in SpaceOAR volume between ultrasound (5.622 cc) and MRI (13.19 cc). Part of this discrepancy arises from the superior resolution and quality of the MRI. Discrepancies also arise from the displacement of anatomy due to the rectal ultrasound probe. The large SpaceOAR volume discrepancy between ultrasound and MRI has no impact on patient care; however, is important to consider.
The dosimetric benefit of increasing prostate and rectal separation is the primary objective of using the SpaceOAR gel during HDR prostate implantations. This can be seen in the craniocaudal direction on the sagittal image (Figure 2) and anteroposterior direction in the axial images (Figure 1 and Figure 3). The average distance between the closest needle and the rectal wall in the past ten HDR prostate procedures at our institution was $3 \mathrm{~mm}$ (median, $2.9 \mathrm{~mm}$; max, $6.3 \mathrm{~mm}$; min, $0.4 \mathrm{~mm}$ ). Utilizing SpaceO$\mathrm{AR}$, the distance between the closest needle and the rectal wall lacking SpaceOAR averaged $6 \mathrm{~mm}$, and the distance

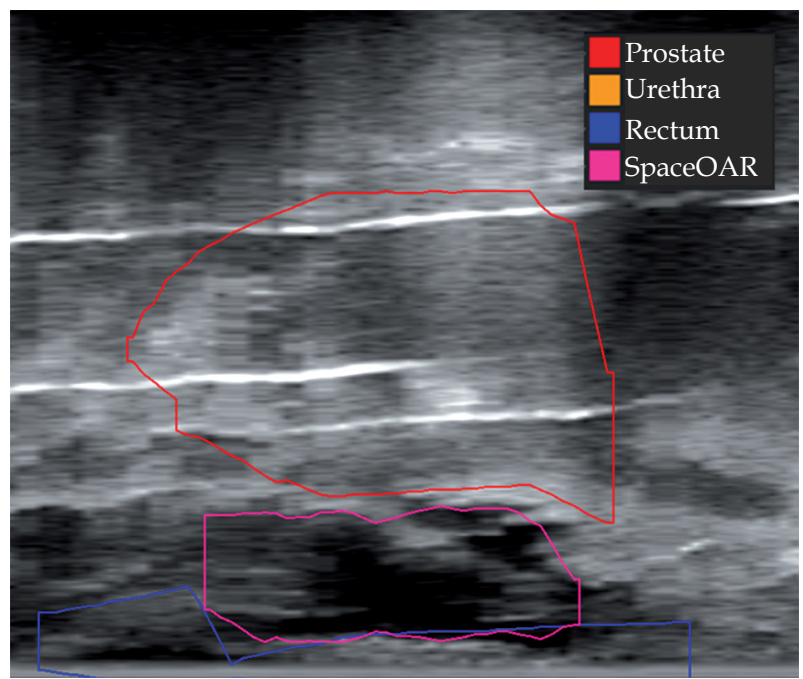

Fig. 2. Sagittal slice from an ultrasound image after complete needle implantation during an HDR brachytherapy prostate procedure post-SpaceOAR injection with overlaid contours. Please note that the poor image quality results from this image's reconstruction from an axially acquired ultrasound and that the contours were generated in the axial view where image quality is adequate. This image is meant for visualization of the gel's craniocaudal displacement of the prostate 


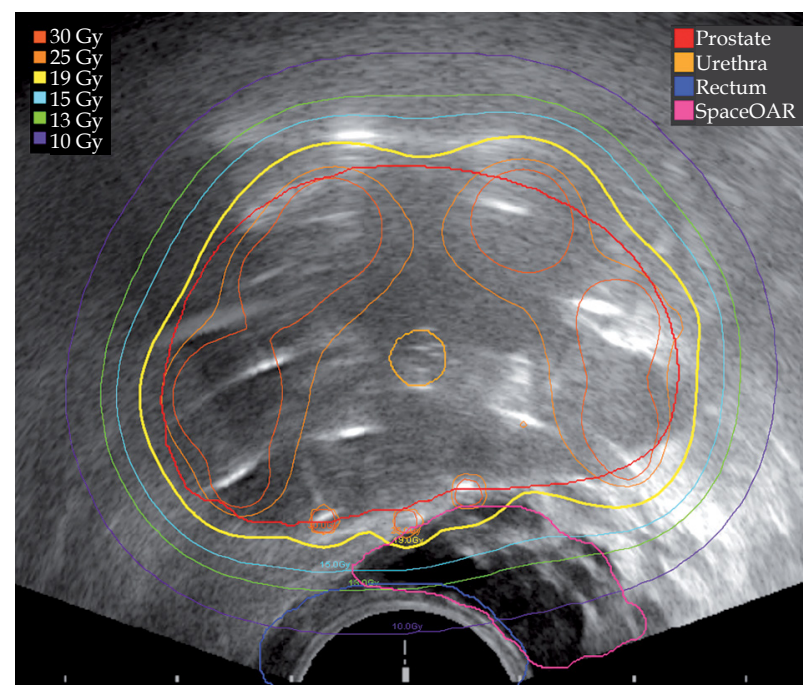

Fig. 3. Isodose distribution and contours from the HDR brachytherapy prostate implant overlaid on the ultrasound image

between a needle within the region of the SpaceOAR and rectal wall was greater than $8 \mathrm{~mm}$. In regions of no SpaceOAR, its contralateral presence increased the prostate-rectum spacing. This increased separation and shift in isodose lines is beneficial due to the large dose gradient obtained with an Ir-192 source (Figure 3). In a phase I study done at our institution to assess the safety, tolerability, and preliminary efficacy of HDR brachytherapy in combination with hypofractionated VMAT in intermediate-risk prostate cancer, the following median dosimetry was reported for 22 patient plans [40]: Prostate $-\mathrm{V}_{100 \%}=96.3 \%$, $\mathrm{V}_{150 \%}=39.4 \%, \mathrm{~V}_{200 \%}=15.3 \%$; Urethra $-\mathrm{V}_{118 \%}=1.39 \%$, $\mathrm{D}_{2 \mathrm{cc}}=71.7 \%$; Rectum $-\mathrm{V}_{75 \%}=1.3 \mathrm{cc}$. This case achieved: Prostate $-\mathrm{V}_{100 \%}=97.3 \%, \mathrm{~V}_{150 \%}=35 \%, \mathrm{~V}_{200 \%}=14.5 \%$; Urethra $-\mathrm{V}_{118 \%}=0 \%$; Rectum $-\mathrm{D}_{2 \mathrm{cc}}=51.6 \%, \mathrm{~V}_{75 \%}=0 \mathrm{cc}$ (Figure 4).

Using a more conservative $\alpha / \beta$ of 3 Gy for rectum, max equivalent dose at $2 \mathrm{~Gy}$ per fraction (EQD2) to $2 \mathrm{cc}$ of rectum was $25.1 \mathrm{~Gy}$, in accordance with GEC-ESTRO recommendations to include a $\mathrm{D}_{2 \mathrm{cc}}$ to rectum less than $75 \mathrm{~Gy}$ by EQD2 [41,42]. SpaceOAR resulted in better dosimetry for all critical dosimetric indices as reported in our phase I trial [40]. QUANTEC guidelines suggest a conservative DVH constraint set for the rectum of $\mathrm{V}_{50 \mathrm{~Gy}}<50 \%, \mathrm{~V}_{60 \mathrm{~Gy}}<$ $35 \%, \mathrm{~V}_{65 \mathrm{~Gy}}<25 \%, \mathrm{~V}_{70 \mathrm{~Gy}}<20 \%$, and $\mathrm{V}_{75 \mathrm{~Gy}}<15 \%$ [3]. As our $\mathrm{D}_{2 \mathrm{cc}}$ rectum was $25.1 \mathrm{~Gy}$ after EQD2 conversion using an $\alpha / \beta$ of 3 for late effects, these dose constraints were easily met. This is likely attributed to an ideal implant in addition to increased prostate-rectum spacing, which allowed the optimizer to effectively utilize posterior catheters.

SpaceOAR did not impede standard procedure times, which is important as the patient is under general anesthesia and complication probabilities increase with increased duration of the procedure. The time from patient intubation to HDR initiation was 211 minutes, which is on par with our average HDR prostate procedure time of 203 minutes taken from eight previous procedures. However, there are many variables contributing to procedure time, which makes it difficult to assess and compare

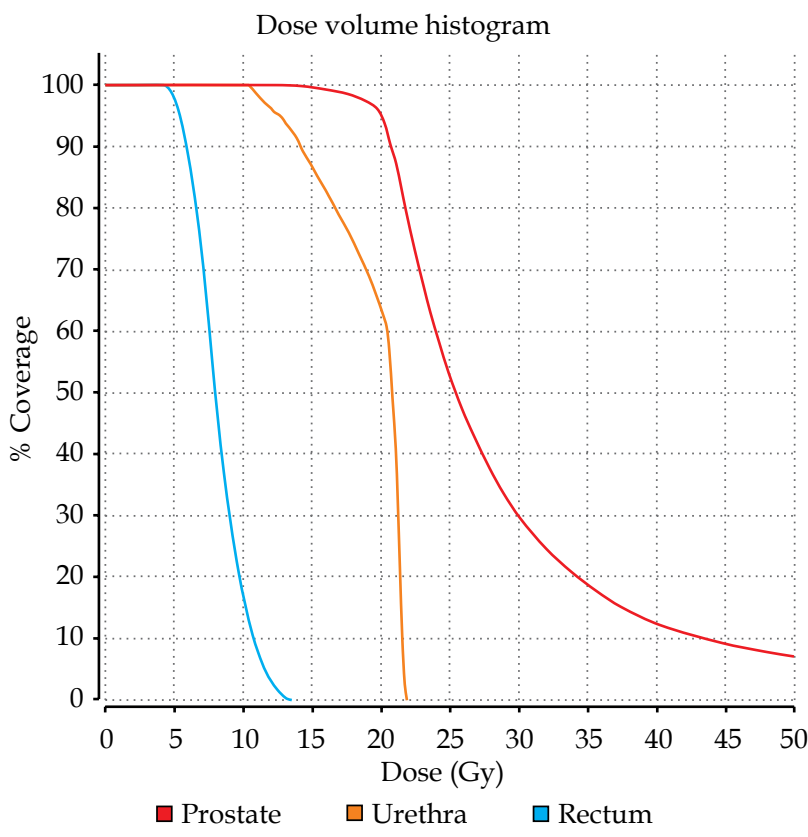

Fig. 4. Dose-volume histogram from the HDR brachytherapy prostate procedure

actual times on a case-by-case basis. Additional patient safety factors created by SpaceOAR are buffer space between needle placement and the rectum, which decreases the probability of rectal perforation. Mariados et al. [30] report no device-related adverse events or rectal perforations during the use of the spacer and discuss ease of use for applying the spacer with $98.7 \%$ placement success.

\section{Conclusions}

SpaceOAR successfully increased catheter-rectal wall spacing and decreased rectal dose due to improved planning capabilities, while decreasing the likelihood of rectal perforation. Although difficult to directly conclude given the lack of prospective randomized series, incorporating techniques to increase prostate-rectal wall separation may be of particular benefit to high-risk patients. This technique is prominent in EBRT and may be of interest in brachytherapy incorporating HDR hypofractionated treatment. This particular case report demonstrates one application of this concept in a patient with UC with favorable dosimetric outcome. The patient tolerated the SpaceOAR injection and HDR brachytherapy procedures well. At five months, one week, and one day follow-up, the patient reported no bowel issues following HDR brachytherapy.

\section{Disclosure}

Authors report no conflict of interest.

\section{References}

1. Litwin MS, Tan HJ. The diagnosis and treatment of prostate cancer. JAMA 2017; 317: 2532-2542.

2. Hamdy FC, Donovan JL, Lane JA et al. 10-year outcomes after monitoring, surgery, or radiotherapy for localized prostate cancer. N Engl J Med 2016; 375: 1415-1424. 
3. Michalski JM, Gay H, Jackson A et al. Radiation dose-volume effects in radiation-induced rectal injury. Int J Radiat Oncol Biol Phys 2010; 76 (Suppl 3): S123-129.

4. Stenmark MH, Conlon ASC, Johnson S et al. Dose to the inferior rectum is strongly associated with patient reported bowel quality of life after radiation therapy for prostate cancer. Radiother Oncol 2014; 110: 291-297.

5. Arcangeli G, Fowler J, Gomellini S et al. Acute and late toxicity in a randomized trial of conventional versus hypofractionated three-dimensional conformal radiotherapy for prostate cancer. Int J Radiat Oncol Biol Phys 2011; 79: 1013-1021.

6. Willett CG, Ooi CJ, Zietman AL et al. Acute and late toxicity of patients with inflammatory bowel disease undergoing irradiation for abdominal and pelvic neoplasms. Int J Radiat Oncol Biol Phys 2000; 46: 995-998.

7. Murphy CT, Heller S, Ruth K et al. Evaluating toxicity from definitive radiation therapy for prostate cancer in men with inflammatory bowel disease: Patient selection and dosimetric parameters with modern treatment techniques. Pract $\mathrm{Ra}$ diat Oncol 2015; 5: e215-222.

8. Annede $\mathrm{P}$, Seisen $\mathrm{T}, \mathrm{Klotz} \mathrm{C}$ et al. Inflammatory bowel diseases activity in patients undergoing pelvic radiation therapy. J Gastrointest Oncol 2017; 8: 173-179.

9. Song DY, Lawrie WT, Abrams RA et al. Acute and late radiotherapy toxicity in patients with inflammatory bowel disease. Int J Radiat Oncol Biol Phys 2001; 51: 455-459.

10. Kuban DA, Tucker SL, Dong L et al. Long-term results of the M. D. Anderson Randomized Dose-Escalation Trial for Prostate Cancer. Int J Radiat Oncol Biol Phys 2008; 70: 67-74.

11. Zietman AL, Bae K, Slater JD et al. Randomized trial comparing conventional-dose with high-dose conformal radiation therapy in early-stage adenocarcinoma of the prostate: Long-term results from Proton Radiation Oncology Group/ American College of Radiology 95-09. J Clin Oncol 2010; 28 . 1106-1111

12. Al-Mamgani A, Heemsbergen WD, Peeters STH et al. Role of intensity-modulated radiotherapy in reducing toxicity in dose escalation for localized prostate cancer. Int J Radiat Oncol Biol Phys 2009; 73: 685-691.

13. Eade TN, Hanlon AL, Horwitz EM et al. What dose of external-beam radiation is high enough for prostate cancer? Int I Radiat Oncol Biol Phys 2007; 68: 682-689.

14. Peeters STH, Heemsbergen WD, Koper PCM et al. Dose-response in radiotherapy for localized prostate cancer: Results of the Dutch multicenter randomized phase III trial comparing 68 Gy of radiotherapy with 78 Gy. J Clin Oncol 2006; 24 : 1990-1996.

15. Beckendorf V, Guerif S, Le Prisé E et al. 70 Gy versus 80 Gy in localized prostate cancer: 5-year results of GETUG 06 randomized trial. Int J Radiat Oncol Biol Phys 2011; 80: 1056-1063.

16. Loblaw A, Liu S, Cheung P. Stereotactic ablative body radiotherapy in patients with prostate cancer. Transl Androl Urol 2018; 7: 330-340.

17. Lee WR, Koontz BF. Moderate hypofractionation for prostate cancer. Transl Androl Urol 2018; 7: 321-329.

18. King CR, Brooks JD, Gill H et al. Long-term outcomes from a prospective trial of stereotactic body radiotherapy for lowrisk prostate cancer. Int J Radiat Oncol Biol Phys 2012; 82: 877882

19. Arcangeli G, Saracino B, Gomellini S et al. A prospective phase III randomized trial of hypofractionation versus conventional fractionation in patients with high-risk prostate cancer. Int I Radiat Oncol Biol Phys 2010; 78: 11-18.

20. Mahal AR, Yu JB. Current and emerging standards in radiation therapy for prostate cancer. Am J Hematol Oncol 2017; 13: 11-15.

21. Lee WR, Dignam JJ, Amin MB et al. Randomized phase III noninferiority study comparing two radiotherapy fraction- ation schedules in patients with low-risk prostate cancer. J Clin Oncol 2016; 34: 2325-2332.

22. Skowronek J. Brachytherapy in the therapy of prostate cancer - an interesting choice. Contemp Oncol 2013; 7: 407-412.

23. Mendez LC, Morton GC. High dose-rate brachytherapy in the treatment of prostate cancer. Transl Androl Urol 2018; 7: 357-370.

24. Georg D, Hopfgartner J, Gòra J et al. Dosimetric considerations to determine the optimal technique for localized prostate cancer among external photon, proton, or carbon-ion therapy and high-dose-rate or low-dose-rate brachytherapy. Int J Radiat Oncol Biol Phys 2014; 88: 7157-7122.

25. Tisseverasinghe SA, Crook JM. The role of salvage brachytherapy for local relapse after external beam radiotherapy for prostate cancer. Transl Androl Urol 2018; 7: 414-435.

26. Rodda S, Tyldesley S, Morris WJ et al. ASCENDE-RT: An analysis of treatment-related morbidity for a randomized trial comparing a low-dose-rate brachytherapy boost with a dose-escalated external beam boost for high- and intermediate-risk prostate cancer. Int J Radiat Oncol Biol Phys 2017; 98: 286-295.

27. Krauss DJ, Ye H, Martinez AA et al. Favorable preliminary outcomes for men with low- and intermediate-risk prostate cancer treated with 19-Gy single-fraction high-dose-rate brachytherapy. Int J Radiat Oncol Biol Phys 2017; 97: 98-106.

28. Prada PJ, Cardenal J, García A et al. High-dose-rate interstitial brachytherapy as monotherapy in one fraction for the treatment of favorable stage prostate cancer: Toxicity and long-term biochemical results. Radiother Oncol 2016; 119: 411-416.

29. Tang Q, Zhao F, Yu X et al. The role of radioprotective spacers in clinical practice: a review. Quant Imaging Med Surg 2018; 8: 514-524.

30. Mariados N, Sylvester J, Shah D et al. Hydrogel spacer prospective multicenter randomized controlled pivotal trial: dosimetric and clinical effects of perirectal spacer application in men undergoing prostate image guided intensity modulated radiation therapy. Int J Radiat Oncol Biol Phys 2015; 92: 971-977.

31. Ruciński A, Bauer J, Campbell P et al. Preclinical investigations towards the first spacer gel application in prostate cancer treatment during particle therapy at HIT. Radiat Oncol 2013; 8: 134.

32. Van Wijk Y, Vanneste BGL, Jochems A et al. Development of an isotoxic decision support system integrating genetic markers of toxicity for the implantation of a rectum spacer. Acta Oncol (Madr) 2018; 57: 1499-1505.

33. Hamstra DA, Mariados N, Sylvester J et al. Continued benefit to rectal separation for prostate radiation therapy: final results of a phase III trial. Int J Radiat Oncol Biol Phys 2017; 97: 976-985.

34. Karsh LI, Gross ET, Pieczonka CM et al. Absorbable hydrogel spacer use in prostate radiotherapy: a comprehensive review of phase 3 clinical trial published data. Urology 2018; 115: 39-44.

35. Prada PJ, Jimenez I, Gonz H et al. High-dose-rate interstitial brachytherapy as monotherapy in one fraction and transperineal hyaluronic acid injection into the perirectal fat for the treatment of favorable stage prostate cancer: Treatment description and preliminary results. Brachytherapy 2012; 11: 105-110.

36. Strom TJ, Wilder RB, Fernandez DC et al. Prostate radiotherapy A dosimetric study of polyethylene glycol hydrogel in 200 prostate cancer patients treated with high-dose rate brachytherapy \pm intensity modulated radiation therapy. $R a-$ diother Oncol 2014; 111: 126-131. 
37. Yeh J, Lehrich B, Tran C et al. Polyethylene glycol hydrogel rectal spacer implantation in patients with prostate cancer undergoing combination high-dose-rate brachytherapy and external beam radiotherapy. Brachytherapy 2016; 15: 283-287.

38. Van Gysen K, Kneebone A, Alfieri F et al. Feasibility of and rectal dosimetry improvement with the use of SpaceOAR® hydrogel for dose-escalated prostate cancer radiotherapy. J Med Imaging Radiat Oncol 2014; 58: 511-516.

39. Hedrick SG, Fagundes M, Case S et al. Validation of rectal sparing throughout the course of proton therapy treatment in prostate cancer patients treated with SpaceOAR®. J Appl Clin Med Phys 2017; 18: 82-89.

40. Williams NL, Rivera A, Hardin MT et al. Phase 1 study of high-dose-rate brachytherapy combined with hypofractionated volumetric modulated arc therapy for intermediate-risk prostate cancer. Int J Radiat Oncol Biol Phys 2015; 93: e207.

41. Hoskin PJ, Colombo A, Henry A et al. GEC/ESTRO recommendations on high dose rate afterloading brachytherapy for localised prostate cancer: An update. Radiother Oncol 2013; 107: 325-332.

42. Brenner DJ. Fractionation and late rectal toxicity. Int J Radiat Oncol Biol Phys 2004; 60: 1013-1015. 